\title{
sciendo
}

Int. J. of Applied Mechanics and Engineering, 2018, vol.23, No.4, pp.989-1004

DOI: 10.2478/ijame-2018-0056

\section{FRACTAL MODEL OF TRANSDERMAL DRUG DELIVERY}

\author{
A. WALICKA* \\ University of Zielona Góra, Faculty of Mechanical Engineering \\ ul. Szafrana 4, 65-516 Zielona Góra, POLAND \\ E-mail: A.Walicka@ijame.uz.zgora.pl \\ B. IWANOWSKA-CHOMIAK \\ University Hospital of Zielona Góra, Oncology Department \\ ul. Zyty 26, 65-046 Zielona Góra, POLAND \\ E-mail: B.Chomiak@op.pl
}

\begin{abstract}
Skin, separating the vital organs of a human body, is a desirable route for drug delivery. However, the intact skin is normally permeable only for drug molecules with a low molecular weight. The stratum corneum (SC), being the outermost layer of the skin and the epidermis being the second - more permeable - layer of the skin, play an essential function in transdermal drug delivery. Physical and chemical methods of skin poration are used to enhance transdermal drug delivery. Each poration leads to an irregular system of pores which are connected with a system of micro-capillaries passing through the epidermis. Both the systems by their irregularity form a fractal porous matrix. Drugs administrated by this matrix can be either suspensions and solutions or creams and gels, therefore they have to be modelled as non-Newtonian fluids.

To analyse the fluid flow through the porous matrix the model of the epidermis is assumed as gobbet-andmortar with the tortuous mortar of variable thickness and after transition from the mortar to the tube one considered classical and fractal capillary flows of selected non-Newtonian fluids.

Fractal expressions for the flow rate, velocity and permeability of fluids flow in a porous matrix are derived based on the fractal properties of the epidermis and capillary model. Each parameter in the proposed expressions does not contain any empirical constant and has a clear physical meaning and the proposed fractal models relate the flow properties of considered fluids with the structural parameters of the epidermis as a porous medium. The presented analytical expressions will help understand some of the physical principles of transdermal drug delivery.
\end{abstract}

Key words: drug delivery, human skin, epidermis, fractal model.

\section{Introduction}

Fractal is a natural phenomenon or geometric set that exhibits a repeating pattern displaying at every scale. It is also known as evolving or expanding geometry [1]. Fractal geometry was popularized by Mandelbrot [2] when he showed that for decreasing unit of measurement the length of a natural coastline does not converge but, instead, increases monotonically. His study on the length of the coastline, being scaledependent, marked the origins of fractal geometry which has now found numerous applications in characterizing and describing disordered phenomena in science and engineering (Mandelbrot [3]). More information on the engineering fractals applications may be found in the monograph [1].

Skin is one of the most readily accessible organs of the body; it separates the vital organs from the outside environment and serves as a protective barrier against physical, chemical and microbial attacks.

\footnotetext{
* To whom correspondence should be addressed
} 
The human skin is composed of three layers, consisting in: Stratum Corneum (SC), Epidermis and Dermis (Fig.1), the outer layer is the SC. The epidermis is put in contact with the dermis by so called dermoepidermis function (as permeable membrane). The dermis is constituted by a connective tissue layer of mesenchymal origin. Finally, the inner layer is represented by the hypodermis, mainly constituted by connective tissue [4-10].

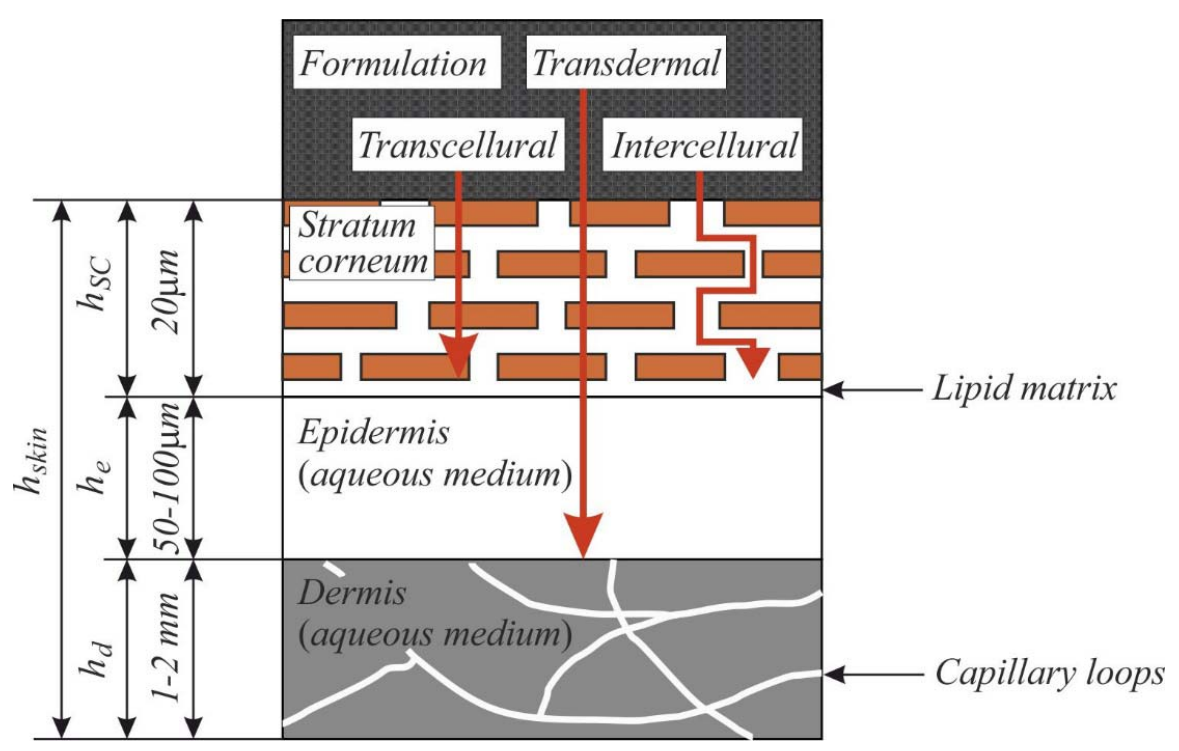

Fig.1. Schematic representation of the transport process involved in drug release from the formulation up to its uptake through the dermal capillaries.

The transdermal drug release is a variable administration route for powerful, not only low-molecular weight therapeutic agents, which has to be precise in their control of drug distribution. This release is specially recommended for many drugs that are difficult to be taken since they must be delivered slowly over a prolonged period to have a beneficial effect [8-10].

The transdermal drug administration has several interesting advantages over other systemic routes; the most interesting is the reduction of first-pass drug degradation as the liver is initially bypassed [5]. The transdermal drug delivery was mainly considered as diffusion transdermal delivery. Recently, methods of drug delivery taking into account the drug flow and the diffusion transport through the skin have been considered. The first application of these methods was described by Siegel [16] who approached insulin delivery by means of an implantable mechano-chemical pump converting changes in blood glucose concentration into a pressure pumping insulin out of the device. This pumping is possible after past execution of micro-capillaries in the skin connected the pump with the dermis, or - more generally - after an enhancement of the skin permeability. While chemical enhancers can be a successful tool for the improvement of small drug permeability through the skin, for big molecules other strategies have to be undertaken. Indeed, proteins and peptides transdermal administration requires the use of physical methods involving electrically based techniques (iontophoresis, electroporation, ultrasound, and photo-mechanical waves), structure-based techniques (microneedles, specially with internal jet-injection), and velocity-based techniques (jet-propulsion) [7-9, 11-15]. Each of these methods leads to the aforementioned execution of micro-capillaries in the skin.

The aim of this paper is to present the drug flow in tortuous capillaries passing through the epidermis layer of the human skin. This flow is analysed twofold: as a classical flow or as a fractal flow. The drugs are modelled as Newtonian fluids (aqueous drug solutions of small molecular weight) or as non-Newtonian fluids (all other drug solutions). 


\section{Structure of the skin after physical poration}

Physical poration of the skin changes temporarily its structure turning the epidermis into a porous layer. Figure 2 shows the hypothetical bilayer poration model involving an electroporation technique [7-9].

Pores are hypothesized to form in the intercellular bilayers via momentary realignment of lipids that recover their original position at various times after an electrical pulse. The distribution of the pores makes a fractal structure.

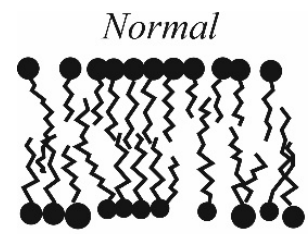

Electroporation

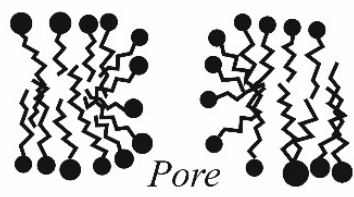

$\mu s-s$ duration
Recovery

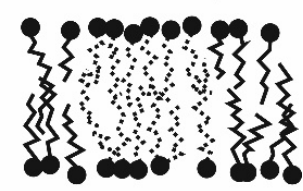

ms-h duration

Fig.2. Hypothetical bilayer poration model.

The pores are connected with a system of micro-capillaries passing through the epidermis. This system may be composed of straight capillaries of constant or variable cross-sections in a transcellular transport or it may be composed of tortuous capillaries also of constant or variable cross-sections in an intercellular transport (Fig.3)

To simplify mathematical considerations, the "gobbet-and-mortar" model of the epidermis is assumed, in which the mortar is tortuous and has a variable thickness (Fig.4).

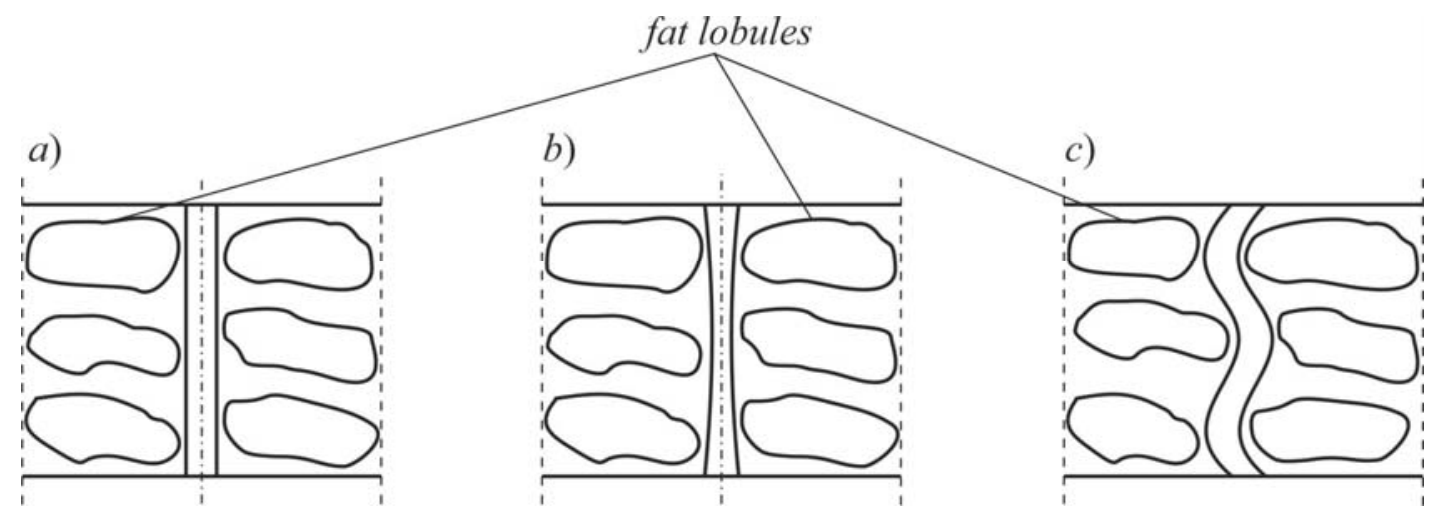

Fig.3. Schematic representation of the geometrical forms of microcapillaries: a) straight capillary of constant cross-section; b) straight capillary of variable cross-section; c) tortuous capillary.

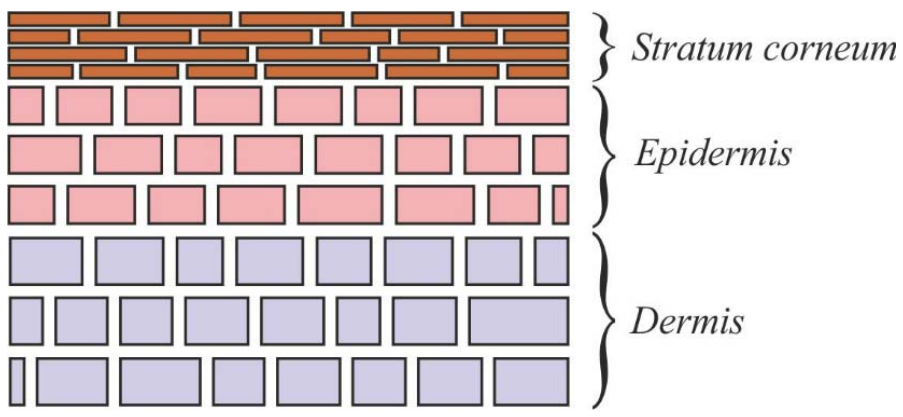

Fig.4. Scheme of a gobbet-and-mortar representation of the epidermis and the dermis. 
Note that the mortar may represent only a capillary fissure. Introducing the notion of a hydraulic radius $R_{h}$

$$
R_{h}=\frac{2 A}{P}
$$

where $A$ is the fissure area and $P$ is the fissure perimeter wetted by a fluid one may pass from the capillary fissure to the capillary tube of radius equal to $R_{h}=r_{c}$.

In further considerations, we will discuss the flows of different fluids in a system of tortuous capillary tubes of variable cross-sections. Introducing the hindrance factor we will be able to model a matrix of capillary tubes of variable cross-sections by a matrix of capillary tubes of constant cross-sections.

\section{Classical flow through the epidermis modelled as a matrix composed of capillary tubes}

We will consider the classical models of flow through the epidermis considered as a matrix composed of capillary tubes. Assuming that the capillaries are tortuous and have variable cross-sections we will present the formulae for the flow of the following fluids: Newtonian, micropolar, pseudoplastic (DeHaven and Sisko models) and viscoplastic (Shulman model). Detailed considerations may be found in [1, 17-20]. These considerations will be supplemented by deliberations on the capillary tortuosity and variability of cross-sections.

Newtonian fluids. Newtonian fluids may model all flows of drugs being aqueous suspension of low molecular weight particles.

The flow velocity through the porous matrix is given as follows (Fig.5)

$$
\mathrm{v}_{N}=\frac{r_{c}^{2} \psi_{N} \varphi_{p}}{8 \mu \tau}\left(-\frac{d p}{d y}\right)
$$

where $r_{c}$ is the maximum capillary radius, $\varphi_{p}$ is the matrix porosity, $\psi_{N}$ is the Newtonian hindrance factor, $\mu$ is the Newtonian viscosity, $\tau$ is the tortuosity (in the case of tortuous capillaries).

The term $d p / d y$ denotes the pressure gradient generated by the donor device.

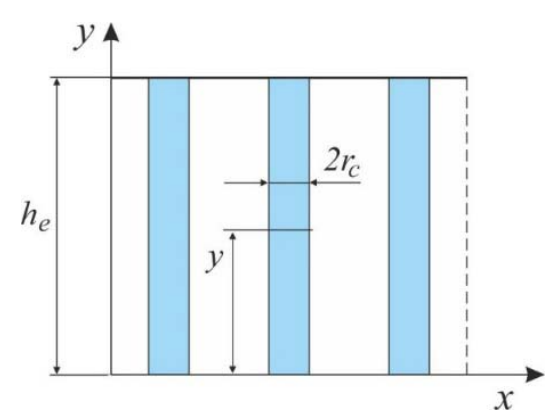

Fig.5. Porous matrix composed of rectilinear parallel capillary tubes.

Micropolar fluid. This fluid represents so called polar fluids which may characterise all drugs being suspensions composed of particles having mean value of molecular weight. These fluids are described by two viscosities: the first of them is similar to the Newtonian shear viscosity $\mu$, the other, $k$, is the vortex (coupling) viscosity describing the particle rotation.

The flow velocity through the porous matrix is given as follows 


$$
v_{m}=\frac{r_{c}^{2} \psi_{m} \varphi_{p}}{8 \mu \tau}\left(\frac{\mu}{\mu+k}\right)\left(-\frac{d p}{d y}\right)
$$

where $\psi_{m} \approx \psi_{N}$ is the hindrance factor, $k$ is the vortex viscosity.

Pseudoplastic fluids. We will consider two essential models of these fluids, namely: DeHaven fluid and Sisko fluid; many well known but more complicated models reduce - for suitably selected material coefficients - either to DeHaven or to Sisko fluids.

The viscosity of a DeHaven fluid displays a non-linear relationship between the shear stress and the shear strain rate. To be more precise, the shear strain rate is a non-linear function of the shear stress. This fluid is characterised by a shear viscosity $\mu$, which is similar to the Newtonian viscosity, material coefficient $k_{i}$ and power exponent $n_{i}$, which represent the aforementioned non-linearity of the shear strain rate.

The flow velocity through the porous matrix is given as follows

$$
\mathrm{v}_{D H}=\frac{r_{c}^{2} \psi_{N} \varphi_{p}}{8 \mu \tau}\left(-\frac{d p}{d y}\right)+\frac{k_{i} r_{c}^{n_{i}+2} \psi_{a} \varphi_{p}}{(2 \tau)^{n_{i}+1} \mu\left(n_{i}+4\right)}\left(-\frac{d p}{d y}\right)^{n_{i}+1}
$$

where $\psi_{a}$ is the second, additional, hindrance factor.

The viscosity of a Sisko fluid displays a non-linear relationship between the shear stress and the shear strain rate. This fluid is characterised by a shear viscosity $\mu$ which is similar to the Newtonian viscosity, an additional viscosity $\mu_{i}$ and power exponent $n_{i}$.

The flow velocity through the porous matrix is given as follows

$$
v_{S}=\frac{r_{c}^{2} \psi_{N} \varphi_{p}}{8 \mu \tau}\left(-\frac{d p}{d y}\right)-\frac{\mu_{i} r_{c}^{n_{i}+2} \psi_{S} \varphi_{p}}{(2 \tau)^{n_{i}+1}\left(n_{i}+4\right) \mu^{n_{i}+2}}\left(-\frac{d p}{d y}\right)^{n_{i}+1}
$$

where $\psi_{S}$ is the second, additional, hindrance factor. Both these models of pseudoplastic fluids render it possible - for properly chosen material parameters - to consider the flows of more complex fluids [1], which may model transdermal delivery of different drug solutions and suspensions.

Note that the use of these models of pseudoplastic fluids requires further studies on the drugs rheology.

Viscoplastic fluids. These fluids may model the flows of different therapeutic substances and agents such as drug suspensions, emulsions, ointments and cremes.

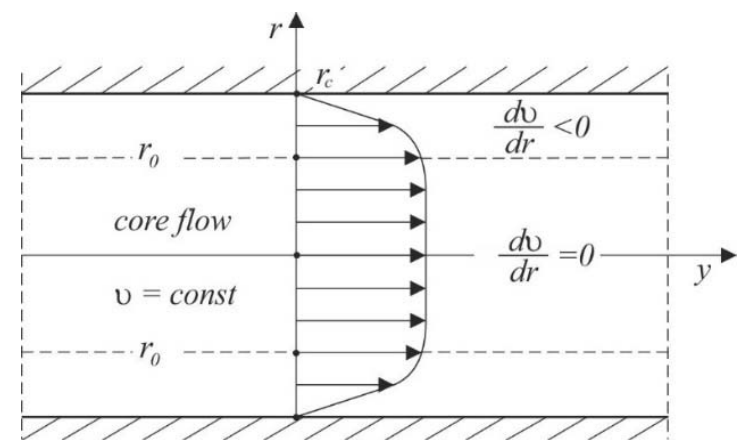

Fig.6. Flow field of a viscoplastic fluid in a capillary tube. 
All these rheological systems are multiphase systems and have many common peculiarities despite different physico-chemical structures which are dependent on the mutual concentrations of phases [22]. One of the common peculiarities is the flow with the yield stress limit characterised by the existence of core flow (Fig.6).

The model of a Shulman fluid is often used to analyse the flows viscoplastic fluids [1]. The full analysis of the flows of viscoplastic fluids described by the Shulman fluid one may find in [1, 17]. The flow velocity of this fluid in a capillary tube of constant cross-section is given as follows [17]

$$
\mathrm{U}=T_{(n)}^{(m)} \frac{\frac{m+n}{r_{c}}}{2^{\frac{m}{n}} \mu}\left(-\frac{d p}{d y}\right)^{\frac{m}{n}}
$$

where: $m, n$ are the flow behaviour indices, $\mu$ is the plastic viscosity and

$$
T_{(n)}^{(m)}=\Upsilon^{\frac{m+3 n}{n}} \sum_{i=0}^{m}(-1)^{i} \frac{n}{m+3 n-i} C_{m}^{i}\left(\Upsilon^{-\frac{m+3 n}{n}}-1\right)
$$

whereas

$$
\Upsilon=\frac{r_{0}}{r_{c}}, \quad C_{m}^{i}=\frac{m !}{i !(m-i) !}
$$

Introducing the hindrance factor $\psi_{S h}$ and the tortuosity factor $\tau$ into (3.5), we may write the following formula for the flow velocity through a porous matrix composed of the capillaries of variable cross-section

$$
\mathrm{v}=T_{(n)}^{(m)} \frac{r_{c}^{\frac{m+n}{n}} \Psi_{S h} \varphi_{p}}{(2 \tau)^{\frac{m}{n}} \mu}\left(-\frac{d p}{d y}\right)^{\frac{m}{n}}
$$

it may be assumed for the rectilinear capillary tubes of variable cross-section that

$$
\psi_{S h}=\left(\psi_{N}\right)^{\frac{m}{n}}
$$

For a special case of a Shulman fluid, when $n=1, r_{0}=0$ (there is no a core flow), we deal with an Ostwald-de Waele (power-law) fluid and then

$$
T_{(1)}^{(m)}=\frac{1}{m+3} .
$$

Note that for flows with a large core, $\Upsilon \approx 1$ and for flows with a small core when $\Upsilon \ll 1$, one can use the following approximations:

- for a large core

$$
T_{(n)}^{(m)} \approx-\Upsilon^{\frac{m+3 n}{n}} \sum_{n}^{m}(-1)^{i} \frac{n}{m+3 n-i} C_{m}^{i}=-\Upsilon^{\frac{m+3 n}{n}} \frac{(-1)^{m}}{3 C_{m+3 n}^{m}},
$$




$$
T_{(n)}^{(n)} \approx-\Upsilon^{4} \sum_{i=0}^{n}(-1)^{i} \frac{n}{4 n-i} C_{n}^{i}=-\Upsilon^{4} \frac{(-1)^{n}}{4 C_{4 n-1}^{n}}
$$

- for a small core

$$
T_{(n)}^{(m)} \approx \frac{n}{m+3 n}\left[1-\frac{m(m+3 n)}{m+3 n-1} \Upsilon^{\frac{1}{n}}\right] .
$$

These formulae are important for all fluids derived from the Shulman fluid, namely: for a Casson fluid $(m=n)$, Vočadlo fluid $(m=1)$, Herschel-Bulkley fluid $(n=1)$ and Bingham fluid $(m=n=1)$.

Note that in medical applications the approximations given by Eqs (3.11) and (3.12) are important only for all $m, n$ being odd numbers $(m, n=1,3, \ldots)$.

\section{Fractal flow through the epidermis modelled as a matrix composed of capillary tubes}

Porous media in nature and also in medicine have been shown to be fractal objectives, and the fractal geometry theory has been proven to be powerful for the flow analysis in these media.

The tortuosity of pores is connected with the fractal properties of the epidermis and it may be defined by a tortuosity factor being the ratio of actual capillary tube length $L_{T}$ to the length (thickness) of the epidermis $L_{0}$. In what follows, we will limit the considerations to the epidermis being a matrix of capillary tubes.

The fractal scaling law for the tortuous capillary is [23-25]

$$
L_{T}=\frac{L_{0}^{T}}{\left(2 r_{c}\right)^{T-1}}
$$

where $T$ is the fractal tortuosity factor.

Differentiating Eq.(4.1), we have

$$
d L_{T}=\frac{T L_{0}^{T-1}}{\left(2 r_{c}\right)^{T-1}} d L_{0}
$$

or passing to $y$ variable

$$
d y_{T}=\frac{T L_{0}^{T-1}}{\left(2 r_{c}\right)^{T-1}} d y .
$$

Therefore the pressure gradient is equal to

$$
\frac{d p}{d y_{T}}=\frac{\left(2 r_{c}\right)^{T-1}}{T L_{0}^{T-1}} \frac{d p}{d y} .
$$


The cumulative size distribution of pores in the porous epidermis also follows the fractal scaling law [1] and is given as [24]

$$
N=\left(\frac{r_{c \max }}{r_{c}}\right)^{F}
$$

where $r_{c \max }$ is the maximum pore (capillary) radius and $F$ is fractal dimension for pores. After differentiation Eq.(4.4) gives

$$
d N=-\frac{F\left(r_{c \max }\right)^{F}}{r_{c}^{F+1}} d r_{c}
$$

it follows from this expression that the number of pores decreases with the increase of pore sizes.

The surface $A$ of the porous epidermis matrix is now equal to

$$
A=\frac{A_{p}}{\phi_{p}}=-\frac{1}{\phi_{p}} \int_{r_{c \min }}^{r_{c \max }} \pi r_{c}^{2} d N=\frac{F \pi r_{c \max }^{2}\left(1-\phi_{p}\right)}{(2-F) \phi_{p}}
$$

where $A_{p}$ is the total pore area,

$$
\phi_{p}=\phi_{r}^{2-F}
$$

is now the fractal porosity [23] and

$$
\phi_{r}=\frac{r_{c \min }}{r_{c \max }} .
$$

\subsection{Fractal model of a Newtonian fluid flow through a porous matrix}

The flow rate $q$ through a single capillary tube of variable cross-section for a Newtonian fluid is given as follows (see also Eq.(3.1))

$$
q\left(r_{c}\right)=\left.\pi r_{c}^{2} v_{N}\right|_{\varphi_{p}=\tau=1}=\frac{\pi r_{c}^{4} \psi_{N}}{8 \mu}\left(-\frac{d p}{d y_{T}}\right)
$$

here the fractal tortuosity of a capillary tube was taken into account.

The total flow rate $Q$ over the matrix cross section can be obtained integrating Eq.(4.9) over the entire interval of pore sizes

$$
Q=-\int_{r_{c \min }}^{r_{c \max }} q\left(r_{c}\right) d N
$$

taking into account Eqs (4.3), (4.5), (4.8) and (4.9) we will obtain 


$$
Q_{N}=\frac{\pi \psi_{N} F r_{c \max }^{3+T}\left(1-\phi_{r}^{3+T-F}\right)}{2^{4-T} \mu h_{e}^{T-1} T(3+T-F)}\left(-\frac{d p}{d y}\right)
$$

here $L_{0}=h_{e}$ is the thickness of the epidermis matrix (see Figs 1 and 5).

Note that the following inequalities hold for the epidermis as the porous matrix

$$
1<T<2, \quad 0<F<2,
$$

then the exponent $3+T-F \geq 2$. In general, $\phi_{r} \approx 10^{-2}$, then $\phi_{r}^{3+T-F}<<1$. Hence Eq.(4.11) can be reduced to

$$
Q_{N}=\frac{\pi \psi_{N} F r_{c \max }^{3+T}}{2^{4-T} \mu h_{e}^{T-1} T(3+T-F)}\left(-\frac{d p}{d y}\right) .
$$

Dividing Eq.(4.12) by Eq.(4.6) gives the average velocity for the flow of a Newtonian fluid through the porous matrix

$$
\mathrm{v}_{N}^{f}=\frac{r_{c \max }^{1+T}(2-F) \psi_{N} \phi_{p}}{2^{4-T} \mu h_{e}^{T-1} T(3+T-F)\left(1-\phi_{p}\right)}\left(-\frac{d p}{d y}\right)
$$

It is easy to see that the fractal flow velocity of a Newtonian fluid through the porous epidermis is more complicated than Eq.(3.1) describing the classical case of this flow. Equation (4.13) is related not only to the characteristic parameter of the fluid such as viscosity $\mu$ but also to the structural parameters of the matrix such as: $h_{e}, r_{c \max }, \phi_{p}, T$ and $F$.

\subsection{Fractal model of a micropolar fluid flow through a porous matrix}

The asymptotic flow rate of a micropolar fluid through a fractal tortuous capillary tube is given as follows

$$
q\left(r_{c}\right)=\frac{\pi r_{c}^{4} \psi_{N}}{8 \mu}\left(\frac{\mu}{\mu+k}\right)\left(-\frac{d p}{d y_{T}}\right)
$$

therefore the total flow rate $Q$ over a matrix cross section will be (see the previous Section)

$$
Q_{m}=\frac{\pi \psi_{N} F r_{c \max }^{3+T}}{2^{4-T} \mu h_{e}^{T-1} T(3+T-F)}\left(\frac{\mu}{\mu+k}\right)\left(-\frac{d p}{d y}\right)
$$

whereas the average flow velocity

$$
\mathrm{v}_{m}^{f}=\frac{r_{c \max }^{1+T}(2-F) \psi_{N} \phi_{p}}{2^{4-T} \mu h_{e}^{T-1} T(3+T-F)\left(1-\phi_{p}\right)}\left(\frac{\mu}{\mu+k}\right)\left(-\frac{d p}{d y}\right) .
$$




\subsection{Fractal model of a DeHaven fluid flow through a porous matrix}

The flow rate $q$ through a simple tortuous capillary of variable cross-section for a DeHaven fluid is as follows

$$
q\left(r_{c}\right)=\frac{\pi r_{c}^{4} \Psi_{N}}{8 \mu}\left(-\frac{d p}{d y_{T}}\right)+\frac{\pi k_{i} r_{c}^{n_{i}+4} \psi_{a}}{2^{n_{i}+1} \mu\left(n_{i}+4\right)}\left(-\frac{d p}{d y_{T}}\right)^{n_{i}+1}
$$

Introducing this equation into Eq.(4.10), after integration, taking into consideration Eqs (4.3), (4.5), (4.8), (4.17), and neglecting the terms of small values, we will obtain the following formula

$$
Q_{D H}=Q_{N}+\frac{\pi \psi_{a} k_{i} F r_{c \max }^{3+\left(n_{i}+1\right) T}}{2^{\left(n_{i}+1\right)(2-T)} \mu\left(n_{i}+4\right) h_{e}^{\left(n_{i}+1\right)(T-1)} T^{n_{i}+1}\left[3+\left(n_{i}+1\right) T-F\right]}\left(-\frac{d p}{d y}\right)^{n_{i}+1} .
$$

Dividing Eq.(4.18) by Eq.(4.6) gives the average velocity for the flow of a DeHaven fluid through the porous matrix

$$
\mathrm{v}_{D H}^{f}=\mathrm{v}_{N}^{f}+\frac{\psi_{a} k_{i} r_{c \max }^{l+\left(n_{i}+1\right) T}(2-F) \phi_{p}}{2^{\left(n_{i}+1\right)(2-T)} \mu\left(n_{i}+4\right) h_{e}^{\left(n_{i}+1\right)(T-1)} T^{n_{i}+1}\left[3+\left(n_{i}+1\right) T-F\right]\left(1-\phi_{p}\right)}\left(-\frac{d p}{d y}\right)^{n_{i}+1}
$$

It is easy to see that the fractal velocity of a DeHaven fluid through the porous matrix is related not only to the structural parameters of the matrix such as: $h_{e}, r_{c \max }, \phi_{p}, T$ and $F$ and the pressure gradient but also to the characteristic parameters of the fluid: $\mu, k_{i}, n_{i}$.

\subsection{Fractal model of a Sisko fluid through a porous matrix}

The flow rate $q$ through a single tortuous capillary of variable cross-section for a Sisko fluid is given by the following formula

$$
q\left(r_{c}\right)=\frac{\pi r_{c}^{4} \Psi_{N}}{8 \mu}\left(-\frac{d p}{d y_{T}}\right)-\frac{\pi \mu_{i} r_{c}^{n_{i}+4} \psi_{S}}{2^{n_{i}+1}\left(n_{i}+4\right) \mu^{n_{i}+2}}\left(-\frac{d p}{d y_{T}}\right)^{n_{i}+1}
$$

Because Eqs (4.17) and (4.20) are similar, we can also deal similarly with the calculations. As a result we have

$$
\begin{gathered}
Q_{S}=Q_{N}-\frac{\pi \psi_{S} \mu_{i} F r_{c \max }^{3+\left(n_{i}+1\right) T}}{2^{\left(n_{i}+1\right)(2-T)} \mu^{n_{i}+2}\left(n_{i}+4\right) h_{e}^{\left(n_{i}+l\right)(T-1)} T^{n_{i}+1}\left[3+\left(n_{i}+1\right) T-F\right]}\left(-\frac{d p}{d y}\right)^{n_{i}+1}, \\
\mathrm{v}_{S}^{f}=\mathrm{v}_{N}^{f}-\frac{\psi_{S} \mu_{i} r_{c \max }^{1+\left(n_{i}+1\right) T}(2-F) \phi_{p}}{2^{\left(n_{i}+1\right)(2-T)} \mu^{n_{i}+2}\left(n_{i}+4\right) h_{e}^{\left(n_{i}+1\right)(T-1)} T^{n_{i}+1}\left[3+\left(n_{i}+1\right) T-F\right]\left(1-\phi_{p}\right)}\left(-\frac{d p}{d y}\right)^{n_{i}+1}
\end{gathered}
$$

It can be seen that in the case of this fluid the velocity depends not only on the structural parameters but on the characteristic parameters of the fluid which are now: $\mu, \mu_{i}, n_{i}$. 


\subsection{Fractal model of a Shulman fluid flow through a porous matrix}

Let us consider at first the flow of a Shulman fluid with a large core through a porous matrix. The asymptotic flow rate $q$ through a single tortuous capillary of variable cross-section is as follows

$$
q\left(r_{c}\right)=-\Upsilon^{\frac{m+3 n}{n}} \frac{(-1)^{m}}{3 C_{m+3 c}^{m}} \frac{\pi r_{c}^{\frac{m+3 n}{n}} \psi_{S h}}{2^{\frac{m}{n}} \mu}\left(-\frac{d p}{d_{y T}}\right)^{\frac{m}{n}} .
$$

Introducing relationships (3.7) $)_{1}$ and (4.3) into the above equation and integrating the result over a matrix cross-section we will obtain the total flow rate

$$
Q=-\frac{(-1)^{m}}{3 C_{m+3 n}^{m}} \frac{\pi \psi_{S h} F r_{c \max }^{3+\frac{m}{n}} T \Upsilon_{\min }^{\frac{m+3 n}{n}}}{2^{\frac{m}{n}(2-T)} T^{\frac{m}{n}} h_{e}^{\frac{m}{n}(T-1)}\left[\frac{m}{n}(T-1)-F\right]}\left(-\frac{d p}{d y}\right)^{\frac{m}{n}}
$$

where

$$
\Upsilon_{\min }=\frac{r_{0}}{r_{c \max }}
$$

Here all small quantities were neglected. Dividing Eq.(4.24) by Eq.(4.6) gives the asymptotic average fractal velocity for the flow of a Shulman fluid with a large core through a porous matrix

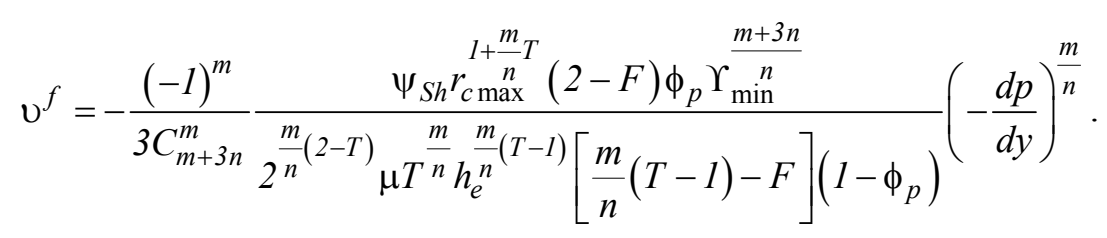

This formula is important for $m$ being an odd number.

Let us now consider the flow of a Shulman fluid with a small core through a porous matrix.

The asymptotic flow rate $q$ through a single tortuous capillary of variable cross-section is given as follows

$$
q\left(r_{c}\right)=\left[1-\frac{m(m+3 n)}{m+3 n-1} \Upsilon^{\frac{1}{n}}\right] \frac{\pi \psi_{S h} n r_{c}^{\frac{m+3 n}{n}}}{2^{\frac{m}{n}}(m+3 n) \mu}\left(-\frac{d p}{d_{y T}}\right)^{\frac{m}{n}} .
$$

Introducing relationships (3.7) $)_{1}$ and (4.3) into the above equation and integrating the result over a matrix cross-section we will obtain the total flow rate

$$
Q=\left[1-\frac{m(m+3 n)\left(3+\frac{m}{n} T-F\right)}{(m+3 n-1)\left(3+\frac{m T-1}{n}-F\right)} \Upsilon_{\min }^{\frac{1}{n}}\right] \frac{\pi \psi_{S h} n F r_{c}^{3+\frac{m}{n}} T}{2^{\frac{m}{n}(2-T)}(m+3 n) \mu T^{\frac{m}{n}} h_{e}^{\frac{m}{n}}(T-1)\left(3+\frac{m}{n} T-F\right)}\left(-\frac{d p}{d y}\right)^{\frac{m}{n}}
$$


Here all small quantities were neglected. Dividing this equation by expression (4.6) gives the fractal average velocity for a Shulman fluid flow with small core through a porous matrix

$$
\begin{aligned}
& \mathrm{v}^{f}=\left[1-\frac{m(m+3 n)\left(3+\frac{m}{n} T-F\right)}{(m+3 n-1)\left(3+\frac{m T-1}{n}-F\right)} \Upsilon_{\text {min }}^{\frac{1}{n}}\right] \times
\end{aligned}
$$

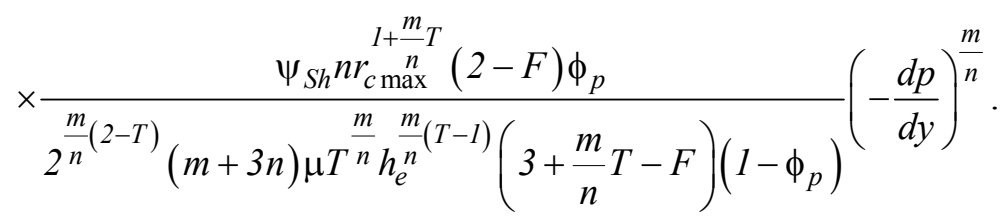

This formula is also important for all fluids derived from a Shulman fluid; note that for the power-law (Ostwald-de Waele) fluid, we have $r_{0}=0\left(\Upsilon=\Upsilon_{\min }=0\right)$ and $n=1$. For a Newtonian fluid $m=n=1, r_{0}=0$ this formula reduces to the form described by Eq.(4.13).

\section{Fractal permeability of the epidermis}

For a sufficiently small porous regions, such as medical scarps of the skin under drug delivery devices, the fluid flow is described by the Darcy law; which - for a Newtonian fluid - has the form [1]

$$
\mathbf{v}=\frac{\Phi_{N}}{\mu}(-\operatorname{grad} p)
$$

where: $\mathbf{v}$ is the velocity vector, $\Phi_{N}$ is the (coefficient of) permeability.

For a one-dimensional flow there is

$$
\mathrm{v}=\frac{\Phi_{N}}{\mu}\left(-\frac{d p}{d y}\right) .
$$

Comparing this formula with Eqs (3.1) and (4.13) we find that:

- for a classical Newtonian flow there is

$$
\Phi_{N}=\frac{r_{c}^{2} \psi_{N} \varphi_{p}}{8 \tau}
$$

- whereas for a fractal Newtonian flow we have

$$
\Phi_{N}^{f}=\frac{r_{c \max }^{1+T}(2-F) \psi_{N} \phi_{p}}{2^{4-T} h_{e}^{T-1} T(3+T-F)\left(1-\phi_{p}\right)}
$$

in the case of a straight capillary the tortuosity factor is equal to $T=1$ and

$$
\Phi_{N}^{f}=\frac{r_{c \max }^{2}(2-F) \psi_{N} \phi_{p}}{8(4-F)\left(1-\phi_{p}\right)} .
$$


It is easy to see that for a tortuous capillary system the fractal permeability of the epidermis depends on its thickness $h_{e}$ and its fractal porosity, whereas for a straight capillary system this permeability only depends on its fractal porosity.

For the viscoplastic fluids described by a Shulman model the generalised form of the Darcy law is given as follows [26]

$$
\mathbf{v}=\frac{\Phi_{S h}}{\mu}(-\operatorname{grad} p)^{\frac{m}{n}}
$$

where: $\Phi_{S h}$ is the epidermis permeability for a Shulman fluid.

For a one-dimensional flow there is

$$
\mathrm{v}=\frac{\Phi_{S h}}{\mu}\left(-\frac{d p}{d y}\right)^{\frac{m}{n}}
$$

Let us only consider the Shulman fluid flow with a small core; the velocity of a classical flow through a porous matrix is given by Eqs (3.8) and (3.13) whereas the velocity for a fractal flow is given by Eq.(4.29). Comparing these equations with Eq.(5.7) we will find

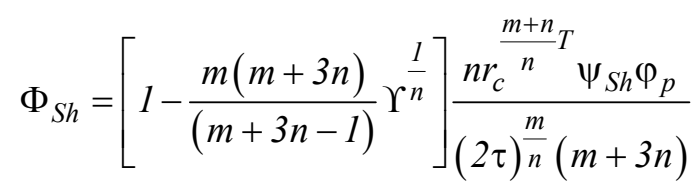

and

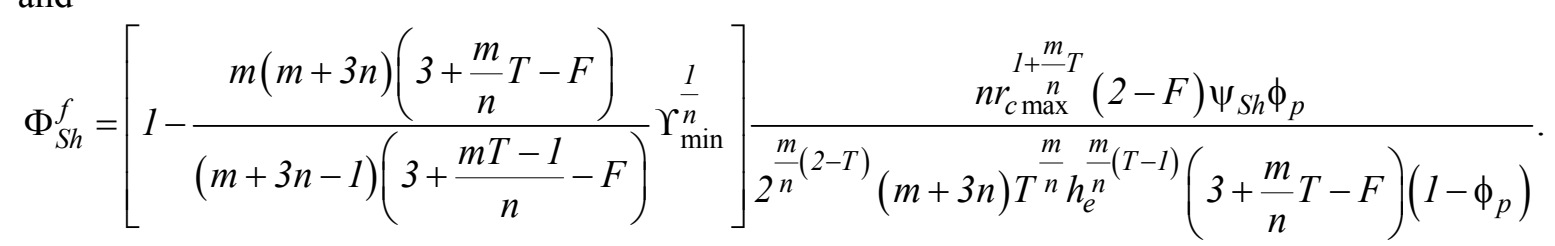

For the system of straight capillaries $(T=1)$ this formula is subject to further simplification.

For the pseudoplastic fluids the generalisation of the Darcy law has the following forms [26]:

- for a DeHaven fluid

$$
\mathbf{v}=\frac{\Phi_{N}}{\mu}(-\operatorname{grad} p)+\frac{\Phi_{D H}}{\mu}(-\operatorname{grad} p)^{n_{i}+1},
$$

in a one-dimensional flow there is

$$
\mathrm{v}=\frac{\Phi_{N}}{\mu}\left(-\frac{d p}{d y}\right)+\frac{\Phi_{D H}}{\mu}\left(-\frac{d p}{d y}\right)^{n_{i}+1}
$$

- for a Sisko fluid

$$
\mathbf{v}=\frac{\Phi_{N}}{\mu}(-\operatorname{grad} p)-\frac{\Phi_{S}}{\mu}(-\operatorname{grad} p)^{n_{i}+1}
$$


and in a one-dimensional flow will be

$$
\mathrm{v}=\frac{\Phi_{N}}{\mu}\left(-\frac{d p}{d y}\right)-\frac{\Phi_{S}}{\mu}\left(-\frac{d p}{d y}\right)^{n_{i}+1}
$$

After a comparison of Eqs (5.11) and (5.13) with Eqs (3.3), (3.4) and Eqs (4.19), (4.22), the epidermis permeability for these two pseudoplastic fluids may be easily found.

\section{Conclusions}

In this paper, we have focused on an analytical description of transdermal drug delivery modelled as flows of different fluids through the epidermis. The epidermis was modelled as a porous layer being a gobbet-and-mortar matrix in which the mortar forms a tortuous capillary of variable thickness.

Introducing a hydraulic radius a capillary fissure may be changed into an equivalent capillary tube. To facilitate the transdermal drug delivery physical poration of the skin is often used. This process leads to the formation of a fractal system of capillary tubes passing through the epidermis to the dermis. These capillaries of variable cross-section are tortuous and they change the epidermis to a fractal porous matrix.

The flow through this matrix was analysed twofold: first, as a classical flow, and next as a fractal flow. Using the formulae for the classical flow we present the new formulae for the fractal flow. An introduction of hindrance factors allow us, in a simple procedure, to take into account the variability of the cross-sections of the capillaries passing through the epidermis.

For the purpose of considering transdermal delivery of drugs in different consistency (e.g.: suspension, gel, cream etc, etc) we presented the flow formulae for various models of fluids.

Based on the theory of fractal geometry, the fractal expressions for the flow rate, flow velocity and fractal permeability for different fluids modelling the drug flow in the epidermis considered as a porous matrix were given. The proposed fractal models are expressed as functions of fluid characteristic parameters, pressure drop and structural parameters of the porous matrix. The results have a clear physical meaning and relate the properties of the fluids under consideration to the structural parameters of the porous matrix.

\section{Nomenclature}

$$
\begin{aligned}
A & - \text { area } \\
A_{p} & - \text { fractal pore area } \\
h & - \text { thickness } \\
h_{d} & - \text { dermis thickness } \\
h_{e} & - \text { epidermis thickness } \\
h_{s c} & - \text { stratum corneum thickness } \\
k & - \text { vortex viscosity } \\
k_{i} & - \text { material coefficient for a DeHaven fluid } \\
L_{0} & - \text { nominal capillary length } \\
L_{T} & - \text { actual (tortuous) capillary length } \\
m_{,} n & - \text { power exponents for Shulman fluids } \\
n_{i} & - \text { power exponents for pseudoplastic fluids } \\
P & - \text { wetted perimeter of a fissure } \\
p & - \text { pressure } \\
Q & - \text { total flow rate } \\
q & - \text { capillary flow rate } \\
R_{h} & - \text { hydraulic radius }
\end{aligned}
$$




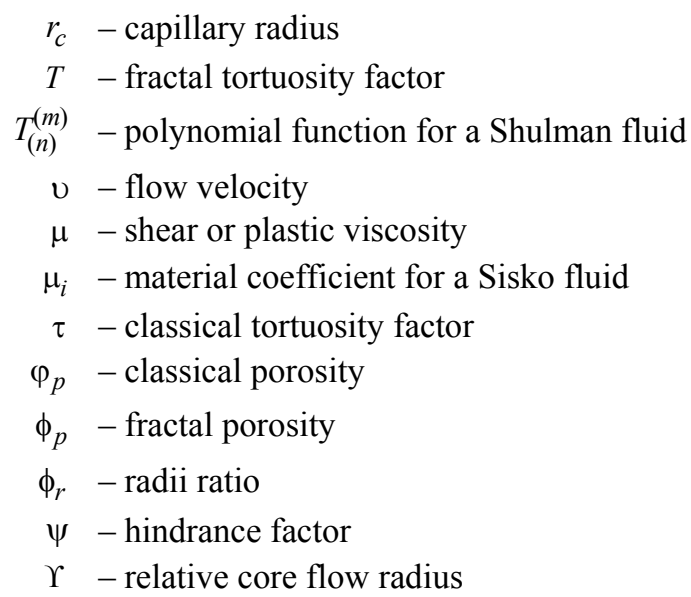

\section{References}

[1] Walicka A. (2017): Rheology of Fluids in Mechanical Engineering. - Zielona Góra: University Press.

[2] Mandelbrot B.B. (1967): How long is the coast of Britain? Statistical self-similarity and fractional dimension. Science, vol.155, pp.636-638.

[3] Mandelbrot B.B. (1982): The Fractal Geometry of Nature. - New York: W.H. Freeman.

[4] Kalia Y.N. and Guy R.H. (2001): Modeling transdermal drug release. - Adv. Drug Delivery Rev., vol.48, pp.159172.

[5] Carreras N., Alonso C., Marti M. and Lis M.J. (2015): Mass transport model through the skin by microencapsulation system. - J. Microencapsulation, vol.32, No.4, pp.358-363.

[6] Milington P.F. and Wilkinson R. (1983): Skin. - Cambridge University Press.

[7] Cross S.E. and Roberts M.S. (2004): Physical enhancement of transdermal drug application: Is delivery technology keeping up with pharmaceutical development? - Curr. Drug Deliv. vol.1. No.1, pp.81-92.

[8] Grassi M. (2008): Membranes in drug delivery. - In: Handbook of Membrane Separations: Chemical, Pharmaceutical, Food and Biotechnological Applications (Pabby A.K., Rizvi S.S.H. and Sastre A.M., Eds), pp.427-464.

[9] Prausnitz M.R. and Langer R. (2008): Transdermal drug delivery. - Nat. Biotechnol., vol.26, No.11, pp.1261-1268.

[10] Anisimov Y.G. and Roberts M.S. (2014): Mathematical models for topical and transdermal drug products. - In: Topical Drug Bioavailability, Bioequivalence and Penetration (Shah V.P., Maibach H.I. and Jenner J., Eds), Second ed., Springer, New York, pp.249-298.

[11] Benson H.A.E. (2005): Transdermal drug delivery: Penetration enhancement techniques. - Curr. Drug. Deliv., vol.2, No.1, pp.23-33.

[13] Arora A., Prausnitz M.R. and Mitragori S. (2007): Micro-scale devices for transdermal drug delivery. - Int. J. Pharm., No.364, pp.227-236.

[14] Cal K. (2009): Across skin barrier; known methods, new performances. - In: Frontiers in Drug Design and Discovery, vol.4 (Caldwell G.W., Ur-Rahman A., Yan Z., Choudhary M.J., Eds), Bentham Science Publisher, New York, pp.162-188.

[15] Cal K. and Stefanowska J. (2010): Methods for skin permeation enhancement of drug substances. - Technology of drug form, vol.66, No.7, pp.514-520.

[16] Siegel R.A. (1990): PH-sensitive gels: Swelling equilibria, kinetics and applications for drug delivery. - In: Pulsed and Self-Regulated Drug Delivery (Kost J. Ed.), CRC Press, New York, pp.129-157. 
[17] Walicka A. (2018a): Simulation of the flow through porous layer composed of converging-diverging capillary fissures and tubes. - Int. J. Appl. Mech. Eng., vol.23, No.1, pp.161-185.

[18] Walicka A. (2018b): Flows of Newtonian and power-law fluids in symmetrically corrugated fissures and tubes. Int. J. Appl. Mech. Eng., vol.23, No.1, pp.187-215.

[19] Walicka A., Walicki E., Jurczak P. and Falicki J. (2018): Effect of hindrance factors on a squeeze film of a porous bearing with a DeHaven fluid. - Submited to: Machine Dynamics Research.

[20] Walicka A., Jurczak P. and Falicki J. (2018): Flows of Sisko fluid through symmetrically curved capillary fissures and tubes. - Submited to: Machine Dynamics Research.

[21] Kushner J., Deen W., Blankshtein D. and Langer R. (2007): First-principles, structure-based transdermal transport model to evaluate lipid partition and diffusion coefficients of hydrophobic permeants soley from stratum corneum permeation experiments. - J. Pharm. Sci., vol.96, No.12, pp.3236-3251.

[22] Ferguson J. and Kembłowski Z. (1991): Applied Fluid Rheology. - London, Elsevier.

[23] Yu B.M. and Li J.H. (2001): Some fractal characters of porous media. - Fractals, vol.9, No.3, pp.365-372.

[24] Yu B.M. (2002): A fractal model for permeability of bi-dispersed porous media. - Int. J. Heat Mass Transfer, vol.45, No.14, pp.2983-2993.

[25] Yu B.M. (2005): Fractal character for tortuous stream tubes in porous media. - Chin. Phys. Lett., vol.22, No.1, pp.158-160.

[26] Walicki E. (2005): Rheodynamics of Slide Bearings Lubrication (in Polish). - Zielona Góra: University Press.

Received: February 16, 2018

Revised: April 25, 2018 